\title{
PARALELISMO, CICLICIDAD Y CREATIVIDAD EN EL ARTE VERBAL MAYA YUCATECO ${ }^{1}$
}

\author{
VAlentiNa Varnarssy \\ CNRS, EREA-I.ESC, Universidad Paris 10
}

\section{Una estabilidad inestable}

Los discursos rituales yucatecos se caracterizan por tal densidad e imbricación de recurrencias de diversos tipos, que procura una sensación de vértigo, nacida de la combinación entre la intensa iteración y una variación que parece infinita. El presente estudio quiere ser una contribución a la definición y la comprensión de los procedimientos retóricos complejos y refinados que fundan estas caracteristicas del arte verbal maya.

Uno de los procedimientos más involucrados es la figura del paralelismo. Varios estudios, algunos ya clásicos, ${ }^{2}$ han recalcado el papel primordial que cumple esta figura en la composición de textos rituales y la de otros géneros discursivos. así como su importancia en tanto base de un principio estético y performativo de las tradiciones orales mayas ( $f$ r. Monod Becquelin y Becquey, en este volumen, para una visión general). Ell paralelismo se encuentra en muchas otras tradiciones donde ha sido en ciertos casos detalladamente estudiado. ${ }^{3}$ Empero, cabe aun mejorar la comprensión de las especificidades de cada lengua y cultura en su uso, que pueden atañer a aspectos tan diversos como el papel de la prosodia, el tipo de unidad lingüistica afectada, o las relaciones semánticas que sustentan y crean los paralelos. El objetivo más preciso del presente articulo es mostrar, a partir del análisis profundo de un caso, la ubicuidad y la variedad de niveles en los cuales se desarrollan los paralelismos en ciertos géneros, donde se multiplican de manera intricada y superpuesta sobre diferentes unidades de composición del

'Una primera versión de este estudio fue presentada en el VIl Congreso Internacional de Mayistas en Mérida, julio 2007. Se integra a un trabajo colectivo realizado por el grupo de imestigación "Pa. ralelismos' en el marco del proyecto 'Géographies du sacré: dynamisme des identités et des espaces mayas" (CNRS, Ministère de la Recherche, Francia).Agradecemos a don Adriano por habernos iniciado y ofrecido un acceso privilegiado a los diälogos analizados, asi conso a don Delio C. C, por su ayuda en la traducción del maya; 2 ambos, por su gran generosidad y paciencia.

${ }^{2}$ Entre ellos: Gossen, 1974; Bricker, 1974; Tedlock, 1983a, 1983b; Haviland, 1992; Breton, 1994; Monod Becquelin, 1979, 1986, 1987; Hanks, 1986, 1959; Hull, 2000; Montemayor, 1999, Fought, 1985; Norman, 1983; Towsend, 1983.

${ }^{3}$ Desde los primeros trabajos de Lowth $(1787)$ sobre la estructura poética en el hebreo de los salmos y el Nuevo Testamento, los de Davis (1830) de la poética china, mis tarde los estudios de la poética europea de Jakobson (1966, 1973), hasta los de fax [1975, 1985] para ladonesia, o de Kumsey (2002) sobre lenguas de Nueva Guinea. 
texto. Una hipótesis es que esta configuración multinivelada podria corresponder a una caracteristica del uso del paralelismo en las tradiciones orales de las diversas lenguas mayas, y quizá más en algunas que en otras. No sólo es preciso ir más allá de la noción de paralelismo binario y contiguo, cualquiera sea la naturaleza de sus constituyentes formales o semánticos, para considerar figuras de recurrencias que abarcan números mayores de elementos, distancias variadas, etc., también cabe interrogarse sobre cómo se construye el texto o discurso en su totalidad, tomando en cuenta otras figuras de composición que funcionan de manera complementaria y a otros niveles que el paralelismo, como lo iniciaron agudamente los estudios de Fought (1985) sobre discursos rituales y cuentos ch'orti, Hanks (1986) sobre textos coloniales yucatecos, o Breton (1994) sobre el Rabinal Achi de los k'iche', y lo plantean Monod Becquelin y Becquey (en este volumen). Siguiendo los pasos de Fought, que ya se interrogaba sobre la relación entre el paralelismo (couplet) y los modelos cíclicos, nuestro propósito es también destacar el papel esencial que cumplen estas otras figuras compositivas, en particular la ciclicidad, en el juego móvil que une de manera indisociable en la tradición estudiada, iteración y variación.

Esta perspectiva siempre nos pareció particularmente importante al analizar los discursos yucatecos de la tradición que conocemos personalmente, más aquella de los descendientes de los rebeldes de la Guerra de Castas, instalados a mediados del siglo xa en la región central del actual estado de Quintana Roo. En efecto, el estudio de los pares léxicos en la oralidad yucateca contemporánea puede conllevar cierta frustración si uno se focaliza en los aludidos pares, sobre todo al compararlos con discursos de otras regiones o épocas. Aqui, los pares léxicos no aparecen con el florecimiento esperado. No es tanto una cuestión de abundancia, porque si estan muy presentes, sino de lo que atañe a la diversidad y creatividad de las asociaciones entre lexemas. Siguiendo la expresión de James Fox, el gran estudioso de los paralelismos en culturas asiaticas, el 'range' (el espectro de términos que pueden asociarse a otro término en paralelismos léxicos) de los paralelismos yucatecos tiende a ser relativamente reducido. ${ }^{4}$ Además, en ciertos géneros como el que analizaremos a continuación, muchos de los pares léxicos provienen del vocabulario religioso introducido por el cristianismo. Si bien esto demuestra la dinámica del procedimiento que transfiguró la retórica de los misioneros, también conlleva una cierta cristalización de los términos asociados en pares. Los textos pueden parecer muy estereotipados, compuestos de expresiones convencionales, generalmente formadas en paralelismos duales o triples, que se repiten casi constantemente.

Un conjunto tal de elementos deberia procurar monotonia, homogeneidad, estatismo. Pero no, todo lo contrario; lo que proporcionan estos textos es una fuerte sensación de dinámica, de estabilidad inestable, de uso inventivo de las

${ }^{4}$ Nos referimes aqui a discursos rituales y no incluimos en particular el habla cotidiana, donde el paralelismo se ejerce aparentemente de manera mis libre. 
convenciones, de estructura con formas imprevisibles, que le otorgan toda su belleza, iCómo explicarlo? Nuestra idea principal es que la dinámica y creatividad recaen al mismo tiempo en juegos y combinaciones variadas entre diferentes formas de recurrencia, y en el principio básico de que aunque todo, o casi todo, se debe repetir, nada debe ser idéntico. Eso, como un principio estético, pero también porque la individualización de cada enunciación es lo que posibilita su eficacia, a nivel interlocutivo y ritual.

En este articulo, desarrollaremos esta hipótesis con el análisis de los diálogos rituales de la peregrinación de San Juan Bautista, santo patrón de X Kopchen, un pueblo maya de Quintana Roo. Cada año, San Juan Bautista $\rightarrow$ mejor dicho, el cayado que lleva en la mano y lo representa, y que ellos llaman bóara, del español "vara'- sale a "pedir la caridad" en un largo recorrido por una docena de pueblos circundantes a X Kopchen, donde es recibido con hartas ofrendas y rezos. En sus llegadas y partidas, pero también en otras ocasiones durante la estadia del santo, los dignatarios encargados de transportar el cayado desde X Kopchen intercambian varios diálogos rituales con quienes lo reciben. Por ellos expresan la transmisión del cargo e indicaciones respecto a los ritos que se han de cumplir, asi como informaciones relativas a acontecimientos previos del trayecto. Para una descripción más precisa de la peregrinación y del contenido de los diálogos, remitimos al lector a Vapnarsky (2000). Aqui nos focalizaremos en los procedimientos de composición y estructuración del discurso, sabiendo que un motivo fundamental para estudiarlos es acceder a una mejor comprensión de la semántica y los valores pragmáticos del texto.

Elegimos estos diälogos por varias razones. En primer lugar, porque han sido poco o nada estudiados, en parte por haber sido considerados muy influidos por el cristianismo y por ello "menos maya". Sin embargo, más alla de la impresionante impregnación del vocabulario y las metáforas introducidas por los misioneros, queremos subrayar la destreza con que los mayas, como en otros casos, tomaron, mejor diriamos aprovecharon estos elementos y los integraron a formas discursivas potentes y versaitiles, propias de su tradición oral y escrita secular, creando con ellos nuevos estilos y géneros. ${ }^{5}$

La otra razón por la cual nos detenemos en estos textos reside en que, en el amplio corpus de discursos recogidos a lo largo de varios años de trabajo de campo, parecen ser aquellos donde se desarrollan de manera más sutil diferentes niveles de estructuración de la recurrencia, asociados a diferentes formas

\footnotetext{
${ }^{5}$ En ese sentido, cabe señalar la distinción clara que existe para los locutores entre el credo y los diallogos. El credo son "rezos" que se aprenden de memoria y casi no sufren variaciones jesea en su versión maya o espanolal. Los dialogos, en cambio, asi como la parte del k'ubik de las palabras rituales la entrega en si misma, el acto discursivo por el cual se invitan o cowocan los seres sagra. dos al altar y se les regalan las ofrendas), son el lugar donde se ejerce la maestria del orador en el uso de ese juego complejo entre repetición y variación; se caracterizan por su gran versatilidad. E credo es coosiderado como una ofrenda, a la par de las ofrendas de comida a las velas, por ejemplo, mientras que los diilogos, asi como el $K$ ubik, son propiamente los motores de la acción ritual tvíase también nota 14).
} 
de variación. Esto se debe probablemente en gran parte a su carácter dialogal y público. Implican una interacción entre dos locutores, dos portadores de las voces de sus pueblos, que tienen que inventar, al correr de sus palabras, una competición solidaria basada en la idea de que las palabras del que contesta deben, por lo menos, aparejarse con las de su interlocutor (Vapnarsky, 2000). Asi los vemos improvisar, partiendo de estructuras comunes, para construir voces que sin perder su singularidad, anhelan ser tan complementarias como los labios que se unen. ${ }^{6}$

En este articulo, mostraremos a partir de tres réplicas seguidas de los diálogos rituales de la peregrinación de San Juan Bautista, estudiadas sucesivamente en las secciones 2,3, y 4 , los modos cada vez más complejos, multinivelados e imbricados, de estructuración del discurso, así como las unidades discursivas que éstos implican. En la última sección, presentaremos una sintesis de los procedimientos discursivos destacados, subrayando ciertas implicaciones de este tipo de estudio para la comprensión de los textos, a nivel de su micro y sobre todo macroestructura, desde una perspectiva estilistica, semántica y pragmática.

\section{K'áat utóoh 'óolal "preguntar sobre el bienestar": La salutación y las bases del aparato retórico}

Empezaremos por analizar la salutación del primer locutor — quien lleva el cayado del santo de pueblo en pueblo- a los representantes del pueblo que llegan a su encuentro (el diálogo tiene habitualmente lugar en el ho'kóah, altar de límite de pueblo). En su salutación (que dura unos 30 segundos), el locutor delinea los principios retóricos básicos del género.

\subsection{Paralelismos contiguos (microparalelismo)}

La primera réplica está construida casi exclusivamente en pares de versos paralelos, al igual que el resto del diálogo, aunque la tercera réplica, analizada más adelante, presenta muchos casos de tripletes. Los paralelismos con unidades léxicas como elemento variante son los más comunes. Este elemento se sitúa más a menudo al fin del verso fla parte que varía en los paralelismos contiguos está se-

\footnotetext{
'Las palabras grabadas, transcritas y estudiadas aqui pravienen de uno de los locutores mis expertos y dotados en ese tipo de arte verbal. Fue el encargado de llevar el santo en su peregrinación durante más de veinte anos, también es oficial de la iglesia maya y ejerce como hmèen. Importa senalar cae existe una prohibición tajante de grabar los diallogos de la peregrinación durante el mismo ritual, in situ, asi como de tomar fotos o videos, incluso para los miembros de li misma coumunidad. Nuestras grabaciones se hicieron de regresa de la peregrinación, en casa de los actores. En ese sentido son una reconstrucción, la que no deja de tener consecuencias importantes. Sin embargo, numerosas observaciones del ritual nos confirman que las procesos descritos aqui soo independientes de este cambio de contexto. En realidad, los locutores tienden a elaborar aún más lo descrito aquí.
} 
ñalada en negritas\}. Pero también los locutores frecuentemente emplean paralelismos que hacen variar unidades gramaticales. Por ejemplo, éstos se observan en los versos 3-4 con la altemancia de determinantes: el posesivo de tercera persona $u$ - (indice personal de la serie $A$ ) en ef verso 3 vs el determinante definido (DET) le en el verso 4. En los versos 9-10, para la forma verbal óora-paj "hacerse hora", el locutor pasa del incompletivo (uc.P) en 9 (señalado con el prefijo $k$-, el índice de persona de la serie $A$, $y$ la desinencia - $-\mathrm{a}(\mathrm{I})$ (Now) que sigue el morfema intransitivo $p$-aj (INTR)) al completivo en $10\langle e l-i j$ que marca completivo en la tercera persona se elide porque el verbo no está en posición final de cláusula; es frecuente que el clitico $h$ que indica completivo esté ausente, como en esta frase). ${ }^{7}$

La gran mayoria de los versos paralelos que componen estos diảogos presentan construcciones morfosintácticas paralelas ("paralelismo gramatical", Jakobson, 1966), y las elipsis, que no son raras, pueden crear nuevos paralelismos gramaticales en conjuntos de versos más amplios. Por ejemplo, la elipsis de uti'il u'uchu $t$ 'para que llegue' en el verso 12 con respecto a 11 , y la de uti'il "para (que)' en 14 con respecto a 13 , crea un paralelismo entre $11+12$ y $13+14$ basado en la presencia de la conjunción uti'il en el primer verso del par y su ausencia en el que le sigue.

1. Ki+ r'àan ti kyum tatào fRespuesta: $k i^{+} \mathrm{t}^{+}$àan $\mathrm{ri}$ kyùum tatdaj

Palabras dulcesisagradas a nuestro señot, padre

2. jo'alibe' tatdoia, así es, padre

3. bey tun u-sadonto asi pues $A 3-\operatorname{san} t o$ ast su santo

4. le sdanto k'in-ii, ber santo dia-vocu el santo dia

5. bix tur ubèli jump'e kuxtàa cóno va una vida

\footnotetext{
${ }^{7}$ Las transcripciones respetan la pronunciación del dialogo, a partir de su grabación. Seguimos las convenciones ortogräficas del alfiabeto oficial para el minya yucateco de 1984, con la ünica diferencia que notamos con un acento grave la vocal larga de tooso bajo. Las transcripciones presentan caracteristicas fonológicas que son de orden dialectal o sociolectal jun registro más coloquial| y conciernen de manera general al habla de la región, como la caida casi sistematica de las $\lambda /$ finales icj. kenta, y no kwxtal "vida", ujo'obi y no uja'obil "sa ano"), o el acortamiento de la vocal rearticulada (v'v) a vocal larga tono alto, o vocal corta (por ejemplo el sufijo de la tercera persona plural -aiob, frecuentemente

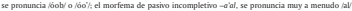
o induso haj. Otras caracteristicas están muche mis ligadas a este zénero de discurso, por ejemplo el alargamiento de ciertas vocales. Con respecto a la segmentación de las palabras, muestras opciones difieren a veces de otras comenciones: unimos los indices personales de la serie A y B a la raiz por considerarlos formas cliticas unimos o no las particulas TAM al verbo según su grado de gramatica. lidad; y juntamos el numeral con los dasificadores numerales, por considerar que ambos forman un tipo de palabra compuesta.
} 
6. Bix tun ubeeli jump'el uyibi tooj 'oblal cómo va una salud consagrada

7. kusitik tech iryumum, que te oftece mi señor

8. kusilik tech injajal ko'lef que te ofrece mi verdadera señora

9. tw'ux k-ny-bora-paj-a men yet int-jojol kolki donde io-A3-hora-1xm-VI por con $A 1$-werdadera señora donde se hace hora por con mi verdadera sef̂ora

10. jebix ka' dora-pàj men yet in-jajal ko'lel, como conj bora-nm por con Al-verdadera sefiora como cuando se hizo hora por con mi verdadera señora

11. uti'if uk'uchu tu 'áanyo para que llegue el año (préstamo del español)

12. Lija'abi, et anิo

13. utitif ali banstéex inywum para que (ustedes) levanten a mi señor

14. abilionskéex injajal ho $\mathrm{lel}$ levanten a mi verdadera señora

15. labalida yan jump'e yîbi kuxtèa, ¿auin hay una vida consagrada?

16. Ibaylíba yan jump'e yibibi tóoj 'óola. ¿aún hay una salud consagrada?

17. kusilik tech iryet injajal boilei xan que te offece mi y mi vesdadera señora también

18. mukaj torapaj men yet injajal ko'lel va a hacerse la hora por y mi verdadera señora

19. ali'boléex yet inyium que se levanten con mi senor

20. (o)lih hoiéex yet injajail) ko'fel xane tatóa. que se levanten con mi verdadera señora también, padre.

\subsection{Recurrencia de pares}

Ciertos pares escanden de manera más continua el discurso, y pueden constituir lo que Hull (2003) llama "framing devices": un emparejamiento común ("common pairing") "that form the backbone of many couplet constructions" (ibid.: 111). Tal es el caso de yium/ jojal ko'lel "señoriverdadera señora" (par que puede referirse a "santo/santa" o a "Jesucristo/Virgen Maria", divinidades de origen cristiano a menudo concebidas en parejaj. Cabe notar que este par también está implicito en la expresión sintética men \# yet injajal ko'lel, por ejemplo en 17 y 18 , donde 
yùum "señor" se omite en la posición \# —elisión muy frecuente en la réplica más larga analizada más adelante- transformando asi un paralelismo (dos versos) en un monástico (un verso). De manera similar a lo señalado para la elipsis, tal reducción permite crear nuevos paralelismos. Aqui, los versos 17 (forma reducida de $7 / 8$ ) y 18 crean juntos un paralelismo basado en el co-texto yet injajal ko'lel "con mi verdadera señora", a la vez que cada uno forma un paralelismo a distancia con $7 / 8$ y $9 / 10$ respectivamente (véase sección siguiente).

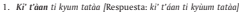
Palabras dulcesisagradas a nuestro señot, padre

2. ja'alibe' tatàúa, así es, padre

3. bey tun usènto así su santo

4. le sabunto k'inii, el santo día

5. bix tur ubeteli jump'e kuxtàa cómo va una vida

6. bix tun ubéeli jump'el uyibi tòoj 'obolal cómo va una una salud consagrada

7. kusilik tech ingưum, que te ofrece mi señot

8. kusitik tech injaja! ko'le! que te ofrece mi verdadera señora

9. tw'ux kizbora paja men yet injajai ko'lel donde se hace hora por con mi verdadera señora

10. jebix ka' iorapaiaj men yet injajal ko iel, como cuando se hizo hora por con mi verdadera señora

11. uti'il uk'uchu tu 'ànyo para que llegue el año (préstamo del español)

12. uja'abi, eไ an̂o

13. uti'if alii banskét inyìmu! para que (ustedes) levanten a mi señor

14. abi'bansketex injajal ko'le! levanten a mi verdadera seniora

15. idoylida yan jump'e yibi kuxtò, ¿aun hay una vida consagrada?

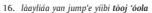
¿aún hay una salud consagrada?

17. kusi ik tech inyest injojol ko lel nort que te ofrece mi y ui verdadera señora también 
18. mukaj doorapoj men yet injajal ko lel va a hacerse hora por y mi verdadera señora

19. abibaleex yet inqụum que se levanten con mi sefior

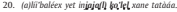

que se levanten con mi verdadera señora también, padre.

\subsection{Composición ciclica}

La organización ciclica, de la cual encontramos descripciones en Fought (1985) para la tradición oral ch'orti, y Hanks (1989) para escritos yucatecos coloniales, implica por lo menos dos conjuntos de versos repetidos en el mismo orden: "discourse is formulated in an ordered series of lines which recur in fixed sequence" (Hanks, ibid: 92, vẻase también Fought, ibid:: 134). Lo más frecuente es que estos versos se construyan por pares de paralelismos contiguos, pero esto no es necesario. ${ }^{5}$ Como veremos mejor a continuación, con ciclos más ricos y complejos, el conjunto de versos incluye generalmente pares de versos que se refieren a "temas" distintos, complementarios y estructurados entre si (por ejemplo, los "temas" pueden ser una referencia temporal — notada Tiempo- o una referencia a acciones del ritual — wookiw- Esto da lugar a una gran diversidad de variaciones de diferente indole, por ejemplo, la omisión o la repetición de un tema, o la creación de paralelismos entre temas de diferentes ciclos (véanse el párrafo siguiente y la sección 3). En esta primera réplica, la ciclicidad se presenta en una forma que consideramos minima, con sólo dos ciclos.

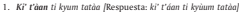
Palabras dulces/sagradas a nuestro senoc, padre

2. jo'alibe' ratdoia, así es, padre

3. bey twi usàento asi su santo

4. le sedonto k'inii, el santo día

5. A) bix tun ubeeli' jump'e kuxtoo como va una vida

6. bux nin ubecli' jump'el uyiabi fòoj 'bolal cómo va una salud consagrada

\footnotetext{
'Hanks (1989. 93) distingue dos tipos de ciclicidad: en el primero, las ciclos están en relación de adyacencia inmediata, mientras que en el segundo, estain separados por material textual. El discurso que analizamos aquí muestra más bien el primer tipo.
} 
7. B) kustilik tech inyùum, que te ofrece mi señor

8. kusitik tech injajal ko Vel que te ofrece mi verdadera señora

9. C) tu'ux kuyeore paja ment yet injajal ho'lef donde se hace bora por con mi verdadera señora

10. jebix ka' borapuoj men yet isjajol ko'lel, Como cuando se hizo hora por con mi verdadera señora

11. D) utlif uk'uchu tu ànyo para que llegue el año

12 . ijiăabi, el periodo

13. E) utial alia'bonskéx inyùum para que (ustedes) levanten a mi señor

14. aîi'banskécx injajal ho'lel levanten a mi verdadera sen̂ora

15. A) Jàabláo yon jump'é yiubi kuxtàa, Laún hay una vida consagrada?

16. Jàayiáo yon jump'e yibi tỏoj 'óola tatin hay una salud consagrada?

17. E) kusrib tech inyet injojol ko lei xort que te ofrece mi y mi verdadera señora también

18 , c) mukaj 'iorapaja men yet injaja' do'lel_ va a hacerse hora por y mi verdadera señora

19 .

20. b) alirbaleex yet inyùm
que se levanten con mi sefiot (a)lif baleex yet injojall) ko7el xane tatabo" que se levanten con mi verdadera sefiora también, padre"

\subsection{Paralelismos entre ciclos}

Se trata ahora de paralelismos entre (pares de) versos que ocupan posiciones equivalentes dentro de la estructura del ciclo. Por ejemplo en la posición $\mathrm{A}$ (subrayado), bix tun ubèeli "Lcómo va?" del ciclo 1 alterna con làalia" yan ("Laün hayitienen?") del ciclo 2. En E, se observa el contraste entre una forma con la conjunción uti'il $\left(E_{1}\right)$ y una forma sin $\left\langle E_{2}\right\rangle$, además del cambio de una forma transitiva alīibonskéex inyium "que hagan levantarse = que levanten" en el primer ciclo, a una forma intransitiva con el ex-objeto presentado ahora en funcion comitativa: alï'baléex yet inyium "que se levanten con", en el segundo cido (pequeñas mayúsculas).

En algunos casos, un verso corresponde a dos versos en el ciclo anterior. Esta figura, que se da en el segundo ciclo para las unidades B y $C$. puede resultar de 
dos procesos en cierta medida opuestos: 1) reducciōn y sintesis de los versos anteriores (caso de B, subrayado con línea de puntos), 2) expansiön del paralelismo del ciclo 1 al ciclo 2 con posible creación de un triplete discontinuo $(1,2 \ldots 3)$. Tal expansión se observa aqui en $C_{1} C_{2}$ (doble subrayado), donde los elementos del paralelismo inter-ciclo que varian crean una progresión aspecto-temporal. Ésta se inicia con una alternancia incompletivo (verso 9/ completivo (verso 10). que permite abarcar el tiempo en su totalidad e infinitud; culmina luego con el uso de una forma de prospectivo inminente (nukaj, verso 18), que ancla el tiempo ritual en el ahora y la singularidad de la enunciación presente.

1. Ki' I'àan $t i k$ kym tafàa [Respuestaz $k i^{\prime} t^{\prime}$ dàn ti kyùm tatdaj]

Palabras dulces/sagradas a nuestro señor, padre

2. ja'alibe' tatdaia', así es, padre

3. bey tan usdanto así su santo

4. le sùento kinii, el santo día

5. A1) bix tun ubèeli jump'e kuxtèa cómo va una vida

6. bix tun ubèeli jump'el uyilibe tòoj "óolal cómo va una salud consagrada

7. Bi) kusile tech inyigum que te ofrece mi señor

8. kusi ik tech injajal ko'le! que te ofrece mi verdadera señora

9. Baunumo (I) tw'ux kwy 'obrapaj men yet injojel ko'le' donde se hace hora por con mi verdadera señora

10. cusumo jebix ka' 'boropiaj nient yet injajal ko'lel, como cuando se hizo hora por con mi verdadera señora

11. Di) uti' uk'uchu to 'èanyo para que llegue el an̂o

12. ujắbì, el axiํา

13. Tewosime

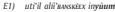
para que (ustedes) levanten a mi señot

14. oili'sucsese injajal ko'lel levanten a mi verdadera señora

15. A2) laegliáa yan junip'e yiubi kuxtòa, counuroverese Laún hay una vida consagrada?

16. làaliáa van jump'e yiabi tóoj 'óola Laun hay una salud consagrada? 
18. FOSP. INLINENTE

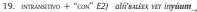
que se levanten con mi señor

20.

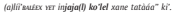
que se levanten con mi verdadera señora también. padre"

\section{Unüuk : "La respuesta" y sus expansiones}

\subsection{Expansion de secciones internas al ciclo}

En su respuesta a la salutación, el dialogante retoma la misma estructura que en el saludo anterior, pero la parte relativa a la acción del ritual es dilatada, con la aparición de nuevas acciones. Éstas están siempre enunciadas en pares y precedidas por el anclaje temporal realizado por la expresión beyjebix ka' 'óorapaja men yet injajall/ ko'lel "como cuando se hizo la hora por y mi verdadera señora". $\mathrm{La}$ sección dilatada tiene la estructura: $\mathrm{C}_{1 D 1 C_{2}} \mathrm{E}_{\mathrm{C}_{3}} \mathrm{D}_{3} \mathrm{E}_{3} \mathrm{C}_{4} \mathrm{E}_{4}{ }^{9}$

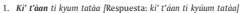
Palabras dulcesisagradas a nuestro señor, padre

2. Mix bik'in bin intus inytum Nunca mentiré a mi señor

3. Mix bik'in bin intus injajal ko'kl Nunca mentiré a mi verdadera sefiora

4. A1) Laayli yon jump'e yibi kuxtàa(I) Aún hay una vida consagrada

5. youn junip'e yiubr tòoj 'úolal hay una salud consagrada

6. BI) kustik tech inyùum, que te ofrece mi señor

7. kusi ik tech injaja(I) ko iel que te ofrece $\mathrm{mi}$ verdadera señora

\footnotetext{
'Los múmeros grandes corresponden a los ciclos textuales, mientras los versos senialados con las cifras en indice corresponden a las expansiones del ciclo. C estai en relación de equivalencia con los otros $C$ aunque no contenga la forma exacta 'odrapajo, porque contiene una expresión con "óona; $y$ la particula bey al inicio çue crea un paralelo con el verso 11 de $C_{Y}$. En realidad $C_{3}$ es una forma mixta

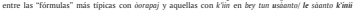
fverso 3,4 del primser saludo).
} 
8. (J) jebix ka' 'borapaj men yet injaja(i) ko'lei como cuando se hizo bora por y mi verdadera señora

9. Di) uk'uchu tu'àaryo que llegue a su año ipréstamo del español)

10. Ir juabi xan, su periodo también

11. C) bey xan 'ooropoj men injaja(l) kolef

Asi tambien se hace hora por mi verdadera señora

12. EJ) kifik'i yet inyum de levantarnos con mi seâor

13. kink'i yet injajal ko lel. de levantamos con mi verdadera señora

14. C) bey tun us shanto 'èroil' asi pues la santa bora

15. D.) taieni' kuthal inyum delante viene mi señor

16.

17.

18. táani kutàal injajal ko'le! delante viene mi verdadera señora ti kpixàan de nuestra alma

19.

20.

22. ri kiv'uma de nuestra tierra E) th bin para dicen kr'ut'Wk ubèel uki' t'àan sàanto káan yet injajal ko'lel que sigamos el camino del bendecido santo cielo con mi verdadera señota t'ùut'l ubéell yiknal jump'el asàanto pixantex que sigamos el camino a la vera de vuestras santas almas

23. C) bey' barapaj men yet injojof bo 'lel sane' inyutum asi se hace hora por con mi verdadera señora también, mi señor

24.

25.

26. E.) kk'ubik jump'e kwenta tinyum que entreguemos un testimonio a nuestro señor kk'ubik jump'e kwertita ta seanto pixan que entreguemos un testimonio a tu santa alma

27. A2) Jasyli yan jump'e kuxtal jump'el amauba lu'une a tu recorrida tierra atis hay una vida 
28. yout jump'e ylïbi thoj 'ólal

hay una salud consagrada

29. B2) kusitik to'on inyet injajal ko'lel xâente' tato.

que nos ofrece mi con mi verdadera señora también, padre.

\subsection{Paralelismo entre réplicas}

Para concluir su respuesta, el segundo locutor retoma versos de una de las preguntas de la salutación que recibió (cfr. $\mathrm{AB}$ arriba), introduciendo ciertas modificaciones. Lo hace mediante un paralelismo que se construye atravesando las réplicas entre dialogantes, y que opera con cambios formalmente minimos pero pragmáticamente esenciales: el paso de una forma interrogativa a una forma asertiva (supresión de la particula interrogativa -ia) y de la segunda persona tech a la primera del plural to'on.

Primer saludo:

(...)

A) Laayláa yon jump'e yỉbi kuxtàal,

interrogación

Laún hay una vida consagrada?

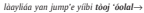

Laun hay una salud consagrada?

B) kastik tech inyet injajal ko'lef xan

2da persona

que te ofrece mi y mi verdadera señora también

Respuesta:

(...)

A) layif- 2 yan jump'e kuxtal

asserción

aún hay una vida

yan jump'e yilbi tỏoj 'óolal

hry una salud consagrada

B) kusilk to'on inyet injajal ko'lel xàne' tataba'

1ra plural

que nos ofrece mi con mi verdadera señora también, padre"

\section{La transmisión del cargo y sus múltiples elaboraciones}

Estos mismos procedimientos rigen la organización de la réplica siguiente que constiturye la parte principal de este diálogo y que es de dimensión, enmarañamiento y sofisticación mucho mayor. Analizaremos ciertas caracteristicas que muestran cómo los recursos mencionados hasta ahora pueden ser usados de manera más compleja. Muestran también la importancia de otros motivos o modos de estructuración del texto, siempre basados en recurrencia con variación. 
Nos concentramos en la primera parte de la réplica, dejando de lado la sección final donde se invocan un conjunto de entidades sagradas terrestres y celestes. La transcripción completa de la réplica, adjunta en anexo, ofrece al lector una visión de su extensión y composición global.

\subsection{Aumento del nuimero de ciclos y formacion de secciones mayores}

Esta réplica también estả compuesta por unidades de distintos niveles. La unidad probablemente principal de estructuración del texto, y que es de nivel intermedio, es la misma unidad ciclica que hemos señalado previamente. Aquí, la estructura básica de la unidad es meMPo-nCC.ós-ESMc.10. El ciclo siempre comienza por una mención con referencia temporal tempo (equivalente a $C$ en las réplicas iniciales), luego viene la mención de Mcaón y la de Espoos. Empero, esta estructura raramente se manifiesta en su forma minima, generalmente es expandida por elementos diversos y segun operaciones variadas, como muestra el cuadro siguiente. Por razones de espacio, sólo nos detendremos en algunos procedimientos, complementarios de los destacados anteriormente. 


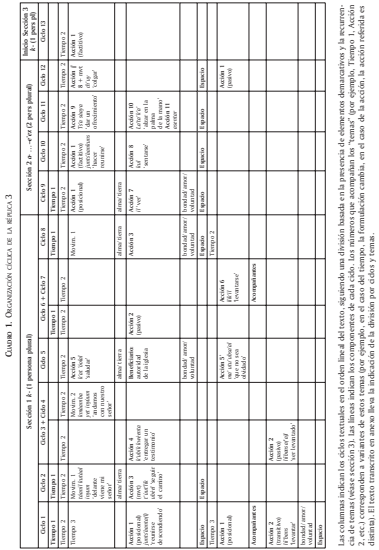




\subsection{Elementos demarcativos de unidad}

Los elementos demarcativos son fundamentales puesto que establecen fronteras entre los ciclos y otras unidades a niveles menores y mayores de construcción del diálogo. Son importantes en la progresión del discurso y de la acción ritual realizada por él, para la memorización, pero también como impulso de nuevos procedimientos retóricos (que se desarrollarán dentro de una nueva unidad, aqui más comúnmente, un nuevo ciclo). En este texto son más frecuentes en la posición inicial del ciclo, pero también se encuentran a menudo en posición final; ${ }^{10}$ pueden corresponder a versos paralelos o formas de monocolon ${ }^{11}$ (véase tambièn Monod Becquelin y Becquey, ibid.).

\section{- frontera de ciclo}

La mención inicial de una o más frases temporales quizá es el elemento más constante del ciclo; parece funcionar como "shifter" de este nivel compositivo."

- frontera de secciones mayores

$\mathrm{La}$ scaón 1 aparece como un tema de límite de secciones (cfir. infra). También TIEMPo 1 tiende a marcar unidades mayores.

\subsection{Fömulas}

Llamamos asi a las expresiones que aparecen repetidamente en el texto pero vienen combinadas con distintos constituyentes del ciclo, acentuando la densidad de la recurrencia. ${ }^{13}$ Por ejemplo, aquí el triplete junp'e 'ủuts / jump'e yáakunah/ jump"e tiibi" "un (gesto) de bondad/ un (gesto de) amor/ un (gesto de) buena voluntad", común a discursos de este género, aparece varias veces en la réplica (ciclos 1, 5, 8 y 9), asociado a distintos elementos. Siempre se integra a una acción que lo precede, pero las acciones varian y los modos de integración son más o menos sintéticos según los casos fpor ejemplo, más sintéticos para el ciclo 8 y menos para el ciclo 1$\}$.

En el ciclo 1:

$$
\text { uti'il uiñ'bansínob utuakli }
$$

para q̨ue eleven su pensamiento

\footnotetext{
"Esto se puede ver por ejemplo en la parte de imvocación de ciertos rezos agricalas donde cada ciclo se conchaye sistemáticamente por la palabra numéceen "mi senooor".

i" Monocolon se puede definir como: "|a| single non parallel line ... which break the narrative flow of parallel structures" (Hull, ibid. 153 ).

12 L única vez que no se menciona el tiempo 2 , éste aparece al final del ciclo (çfi, unidad 8 ).

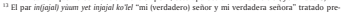
viamente puede analizarse como un caso de fórmula.
} 


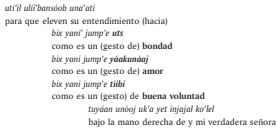

En el ciclo 8:

\author{
ti' kt'utu'lik ubeli le jump'e 'üuts \\ para seguir el camino de un (gesto de) bondad \\ ti' kt'ùut'lik ubéeli jamp'e yóokunaj \\ para seguir el camino de un (gesto de) amor \\ ti' kt'àu'lik ubeeli jump'e tiibi \\ para seguir el camino de un (gesto de) buena voluntad \\ way tuki' t'áan sàanto kàaj yet injajal ko lel \\ aqui en el santo pueblo y mi verdadera Señora
}

\title{
4.4. Expansiones
}

En esta parte del diálogo, el locutor usa un amplio abanico de formas de expandir y dilatar las unidades de su discurso.

- Adición de componentes de los temas del ciclo y creación

de paralelismos contiguos

La creación de tales paralelismos mediante la adición de componentes de temas se da por ejemplo en el ciclo 1. El ciclo empieza con la estructura básica, pero el tema тівия está expresado no por una, sino por tres fórmulas en pares, la tercera siendo menos frecuente (2-7). Lo mismo se da con la expresión de espucio (9-11).

- Creación de paralelismos entre unidades temáticas dentro de un ciclo Luego el locutor retoma la parte tienpo 3, ncoós 1, Espoo, en un gran paralelismo que opera sobre unidades temáticas enteras y donde tieuso 3 , Mcoón 1 están repetidos y crompuintrs viene a formar un par con Espros:

TIEMPO 3, ACOXNN 1, ESPACIO,

TIEMPO 3, AOCOXN 1, ACOMPNNANTES 
(Versos 6-16)

D: uti bis uk'uchu tu'àanyoi

пемIO 3

para que llegue su âิo (prestamo del español)

vja'abi

sแ añ⿰

E. ati'il ujurts'banta jump'e kthata'o'

accoón 1

para que sean reunidos unos padres dignararios

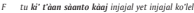

Exap

en el bendecido santo pueblo de mi verdadero y mi verdadera señora

tur ni' yok yet injojol ko' iel

en la punta del pie de y mi verdadera sefiora

tu kíichkelent seanto 'igléesya yet injajal ko'lele'

en la hermosa santa iglesia de y mi verdadera señora

D: k'uch tu 'éanyov

TiEMO 3

llegó a su ofio (préstamo del espanol)

ja'abi

su año

E. uti'il ujunts'banta jump'e ktdata' $a$ '

accoón 1

para que se reúnan nuestros padres/dignatarios

c

yeete jump'e ktanto yun liputàdo acominsiartes
con nuestros padres sefiores diputados
yéete jump'e kraato yun ts'íulobe'
con nuestros padres señores patrones

- Multiplicación de las acciones

Por fin, el locutor expande el mismo ciclo 1 añadiendo otra acción, introducida por la conjunción de finalidad uti'illi). Esta adición es una expansión común de un ciclo que frecuentemente contiene más de una acción. Las segundas y demás acciones pueden correlacionarse de diversas maneras con respecto a la primera [co-referencia, complementariedad, metonimia...), en todo caso están casi siempre introducidas por la conjunción utiil lo que, aunque el co-texto sea minimo, aumenta la relación de paralelismo formal (y sintáctico aqui) entre ellas.

- Despliegue de ciclos en miniciclos o ciclos truncados

Otras veces, la estructura se presenta en forma más simple que la estructura básica. No por ello debe considerársela como más básica; sino más bien, su estatus es el de una forma truncada (o "miniciclo"). Como se puede observar en el cuadro anterior, las formas truncadas corresponden a menudo al despliegue en dos (o más) unidades "truncadas" de una unidad ciclica completa. Este procedimiento se presenta con las unidades (o miniciclos) 3 y 4,6 y $7 .^{14}$

\footnotetext{
${ }^{14}$ En cierta medida este procedimiento se aparenta al que se da en unidades menores y paralelismos contiguos con la elipsis formisión de parte del segundo verso del paralelismot. Sin embargo,
} 
Asi, el ciclo 3 sólo integra tiempo 2, Mcción 4 y scoón 2. La scoós 4 en realidad es equivalente a la accoón 5 del ciclo 5; se refiere al acto de informar a las personas que acompañan el santo durante su trayecto de lo que ocurrió hasta ese momento en el transcurso de la peregrinación, en particular si se percibieron algunos eventos que pudieran ser interpretados como señas divinas. La Acaón 2 ya fue mencionada en el ciclo 1 . Lo que no aparece en el ciclo 3 es la mención de espuco. Observemos ahora el ciclo 4 siguiente: éste sólo incluye nespo 2 y

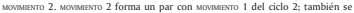
vuelve a mencionar en el ciclo 5 con la expresión amamba lu'uma "sus recorridas tierras" y puede considerarse como equivalente de la ubicación espacial Esmcio. Sumando asi los elementos de las unidades 3 y 4 obtenemos el esquema de un ciclo entero, con correspondencias especificas entre los "miniciclos" fo "ciclos truncados") y los ciclos "enteros" circundantes.

Los ciclos siguientes presentan relaciones similares: a uno bastante extenso, el ciclo 5 , le siguen dos "miniciclos", el 6 y el 7 , que se complementan (el 6 aportando la acción y el 7 el movimiento y los acompañantes).

Esta organización se basa asi en la alternancia de ciclos extensos y ciclos truncados pero unidos por pares: Ciclo $1 /$ Ciclo $2 /$ ciclo $3+4 /$ Ciclo $5 /$ Ciclo $6+7$.

\subsection{Combinaciön de ciclos en unidades mayores (secciones)}

Los ciclos pueden combinarse en unidades mayores; éstas corresponden generalmente a fases destacadas de la acción ritual. En la réplica estudiada, el ciclo 9 corresponde asi a una transición importante. Mientras que en los ciclos anteriores, el locutor siempre habló usando la primera persona del plural para describir lo que él y sus gentes hicieron, hacen o deben hacer, a partir de este ciclo, pasa a la segunda del plural, para indicar ahora a su interlocutores - los dignatarios y habitantes del pueblo que representan- las acciones que les incumbe realizarEl ciclo 9 que inicia esta segunda parte de la réplica retoma los elementos del ciclo 1, en particular la snoón 1. Los ciclos siguientes serán más breves, pero

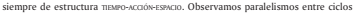
similares a los de la primera sección de la réplica. Por ejemplo, la acción del ciclo 12 retoma y se empareja con la Arociow 8 del ciclo 10 .

En un juego de espejo, la segunda sección se concluye con la primera acción de la réplica "reunir(se) descendiendo", término que se refiere al acto de invocación, acción principal de toda la última parte de la réplica donde se invitan los seres sagrados al altar (versos 107 a 243). El ciclo 12 constituye asi el viraje hacia esa última extensa sección, que se inicia propiamente con el ciclo 13. Además,

mientras que ex los paralelismos contiguos, la elipsis afecta comúnmente la parte inicial del segundo verso ipor ej. en los versas 6,7 o 44, 45 de la réplica 3 en anexo, véase también 2.1 . $;$ en los ciclos truncados, la parte amitida es una porción interna o foal de la unidad considerada (aqui un ciclo completal. 
ya señalamos anteriormente que la scaón 1 funciona como una acción de limite de secciones mayores.

La tercera sección, que no trataremos aqui, está también organizada cíclicamente. Sus ciclos se inician por la misma expresión demarcativa que los anteriores: una frase con óorapajafi) "hacerse hora" (= llegar ef tiempo). Luego, se componen de la mención de los santos y seres sagrados invitados al altar más el lugar en donde están ubicados y se los va a buscar. Los ciclos se articulan entorno a esta referencia espacial puesto que cada uno puede incluir varios seres (mencionados en monásticos, paralelismos de dos, tres o cuatro elementos, o listas) pero sólo un lugar (sea éste un "pueblo santo", es decir uno de los centros ceremoniales de la región, o un sitio más lejano como Jerusalén y la Gloria). Se observan menos variaciones estructurales entre ciclos en esta parte final que en las secciones previas, aunque ninguno es exactamente idéntico a los demás, como se da asimismo en invocaciones de otros géneros rituales.

\subsection{Paralelismos a distancia con desarrollo (progresion y sintesis) de un componente de ciclo a lo largo de toda la réplica}

Indicaremos un último recurso que muestra cómo el paralelismo opera no sólo a nivel de versos contiguos, de pares de versos o entre ciclos sino al nivel de la unidad máxima que representa la réplica entera. Para ello, cabe seguir a lo largo de esta réplica los distintos avatares de la Mccoón 1 junts'an, palabra de la retórica ritual que significa "reunir(se) descendiendo, asentar(se) reuniendo(se) (al altar)". ${ }^{5}$ Esta acción nos muestra, por un juego de paralelismos sobre elementos gramaticales entre versos situados a distintos momentos claves del texto, una progresión clara en la forma de presentar a los participantes en la ceremonia, que contribuye a efectuar la transmisión de cargo y responsabilidad del santo. objetivo central del ritual.

\footnotetext{
${ }^{15}$ Esta palabra es muy usada en los enunciados de entrega (k'ubikj de ofrendas, tanto en rituales relacionados con la iglesia y los santos como en rituales agricolas. Expresa la acción de irwocar e inwitar a los seres sagrados a llegar al lugar del altar para consumir las nfrendas, sean estos seres yuintsib'ab (guardianes del monte), santos y santas, o pixano'ob (almas de los muertos). Es intere. sante que la misma palabra se use aqui para invitar a las personas presentes en el ritual. En todos los casos, la idea principal es que con esa comocación se configura el espacio-tiempo del ritual, los destinatarios invisibles (pero so imperceptibles) o no, ocupando sus kugares definidos en torno al altar. Las frases con àorapaj "hacerse hora", que crean el tiempo del ritual, contribuyen también de manera esencial a esa configuración.
} 
Análisis de las ocurrencias de junts'óan:

cido 1: inicio rêplica, inicio sección 1

\begin{tabular}{|c|c|}
\hline $\begin{array}{l}\text { forma } \\
\text { posicional } \\
\text { intr }\end{array}$ & 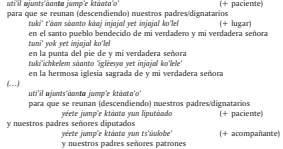 \\
\hline
\end{tabular}

ciclo 9: inicio parte 2

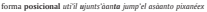
intr

para que se reünan (descendiendo) vuestras santas almas

|+ paciente

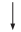

jump'el ominmba fu'monoléex viestras tierras recorridas

cido10. parte 2

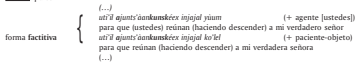

cido 12: conclusiōon réplica (retoma posicional + factitiva: sintesis del texto) 1+paciente)

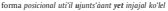

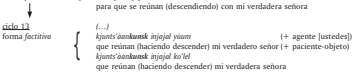

Néanse también los versos $115-116,165$ y 170 -

La palabra junts'an "reunir(se) descendiendo" se menciona ya por primera vez en el ciclo inicial de la réplica, en una forma posicional intransitiva (sufijo-ta(l)). Luego, el locutor la vuelve a usar con la misma forma al inicio de la segunda sección de la réplica, lo que contribuye probablemente a delimitar esta sección. Sin embargo, en el ciclo siguiente, junts'an se usa como verbo transitivo (con el 
sufijo factitivo kun-s) con sujeto segunda persona del plural (a-...-e'ex). Mediante tal construcción, los interlocutores son ahora presentados como sujetos agentes y las divinidades, como objeto de la acción. Este cambio gramatical encarna discursivamente el objetivo de la pasación de cargo: de participantes periféricos, los interlocutores se vuelven agentes de la acción ritual. Por último, en la transición a la siguiente sección, el locutor retoma las dos perspectivas, expresadas en el nivel gramatical por las construcciones posicional y factitiva. Sintetiza asi la progresión que se realizó, a lo largo de la réplica, en la función asignada a los participantes. Cabe notar que el desarrollo de un paralelismo, con una progresión distribuida a lo largo del texto, seguida por paralelismos contiguos que sintetizan los elementos de la progresión, es un procedimiento retórico que encontramos para la expresión de cambios modales o aspecto-temporales en otros géneros discursivos yucatecos (cfr. Vapnarsky 1999, en prensa).

\section{Lo que da vida al texto}

Al término del anälisis de un extracto representativo de los diälogos rituales yucatecos, se destaca la variedad de niveles y procedimientos de construcción discursiva.

En cuanto a niveles, observamos unidades léxicas y gramaticales, versos, fórmulas, tema de ciclo, ciclos, secciones, réplicas. En cuanto a procedimientos, se explicitaron:

- Paralelismos contiguos (con su parte variable formada por elementos léxicos, gramaticales, prosódicos\}

- Paralelismos a distancia entre unidades: temas de un ciclo, ciclos, secciones, réplicas

- Recurrencia y ecos de ciertos pares (leitmotiv o fórmulas)

- Organización ciclica (combinación recurrente y ordenada de temas)

- Expansiones de ciclos (dilataciones, paralelismos contiguos y mega-paralelismo, adición y multiplicación de temas, despliegue)

- Reducción y sintesis de paralelismos y de ciclos

- Organización en secciones (combinación de ciclos con paralelismos marcando fronteras)

- Versos fuera de recurrencia ('monocolon', 'verso aislado')

- listas

Estos procedimientos en realidad están basados en figuras fundamentales en nümero restringido pero de poder extremadamente generativo: leitmotiv, paralelismo, ciclicidad, reducciones y multiplicaciones, ruptura. Tales procedimientos están en correlación con contornos prosódicos y ritmicos, que no hemos podido estudiar aqui. Sobre todo, se articulan con elementos y relaciones semanticas muy diversas, lo que multiplica las significaciones y los efectos que inducen.

Cabe subrayar que la lista previa no pretende ser exhaustiva ni competir con otras tipologias del paralelismo, como aquellas desarrolladas recientemente en 
los fértiles trabajos de Christenson (2000) y de Hull (2003), con los que en muchos aspectos coincidimos. Más bien, en complemento a análisis previos, hemos querido mostrar que los paralelismos se pueden desarrollar en elementos discontinuos a través y a lo largo del discurso, según encadenamientos diversos, y abarcar elementos mucho mayores que los versos. Desde ese punto de vista, la ciclicidad resalta como una figura estructurante esencial para el despliegue de paralelismos entre unidades distanciadas. Lowthe escribia, a propósito de la poesia hebrea antigua, "the several sorts of parallels are perpetually mixed with one another; and this mixture gives a variety and beauty to the composition" (citado por Jakobson, 1966: 400). Los tipos de paralelismos a los cuales se referia implicaban diferentes relaciones entre paralelismos contiguos (sinonimicas, antitéticas, y sintéticas - es decir gramaticales). Un principio equivalente de incesante combinación y recombinación de tipos distintos de paralelismos vale para la tradición maya estudiada. Pero su refinamiento es el de abarcar a la vez, no sólo otras formas de relación, sino también una pluralidad de niveles micro y macrodiscursivos donde se despliegan y se entrecruzan continuamente los paralelos. Esto confirma que los paralelismos, y otros procedimientos retóricos, se deben considerar no sólo como elementos estéticos o poéticos, con sus importantes efectos emocionales y enfáticos (Hull, 2003), sino también como procesos de organización macrodiscursiva, a nivel temático, semántico y pragmático.

Asi, aunque no nos hayamos detenido en el significado preciso de los diallogos analizados, explicitar y tomar en cuenta su organización formal aporta claves esenciales para entender su contenido, como el de todo otro discurso. Las relaciones (de equivalencia, complementariedad, causalidad, etc.) entre las acciones, la función de los anclajes espacio-temporales, las transformaciones epistémicas, el cambio de papel de los participantes, son, entre otros, aspectos centrales de la ceremonia en este caso particular, y del vivir y del actuar en general, que los procedimientos "retóricos" (pero no sólo "retónicos") del hablar determinan de manera central. Este punto también se puede ilustrar volviendo brevemente a la función de la composición cíclica.

La composición ciclica de un texto ha sido relacionada con la referencia del discurso a un recorrido. Hanks (1987) lo propuso acerca de los Deslindes de Yaxcucul y lo hemos desarrollado sobre varios otros textos como el Chilan Balam, el Rabinal Achi, textos mito-históricos de nuestra región de estudio fsobre divinidades que recorren el espacio o el territorio). y también invocaciones de rituales agricolas, donde la voz del h-mëen se desplaza para ir a buscar a los yiuntsilo"ob, guardianes del monte (Vapnarsky, 2001, 2003, Vapnarsky y LeGuen, 2006). En todos estos casos, la ciclicidad textual aparece como una representación icónica del recorrido, y contribuye al carácter espacio-temporalmente instaurador de éste. En los diảlogos de la peregrinación, aunque pueda parecer obvia la imagen del recorrido (la peregrinación en si), cabe subrayar que no se observa el mismo tipo de descripción icónica que en los demás textos mencionados. Los diálogos se integran a un ritual basado en el modelo del recorrido-circuito con altos, pero 
no describen tal recorrido. Parece necesario entonces interrogarse nuevamente sobre el papel de la ciclicidad discursiva en estos diálogos: ¿serả una expresión metafórica del recorrido ritual? iUn procedimiento retórico más amplio, independiente de la imagen del circuito? Esa ciclicidad discursiva estaria subsumida a un modelo cognitivo general de los rituales mayas yucatecos, que implica de una forma u otra la imagen del recorrido, expresada sea gestual y corporalmente, sea discursivamente, sea por ambos u otros medios? 20 un modelo conceptual holistico del tipo que proponia Fought, poniendo en relación la ciclicidad textual con el papel prominente de la figura ciclica en las concepciones mayas del tiempo $?^{16}$ Son unas de las muchas preguntas que surgen de este tipo de estudio y los motivan.

Los procedimientos discursivos explicitados forman los principios de construcción y estructuración del texto, y contribuyen a su definición genérica. Pero a la vez, estos mismos recursos son usados para introducir variación. Como lo señalaba Hanks (1989) al contrastar la organización retórica de narraciones Yupik (Woodbury, 1985) con aquella de los escritos yucatecos coloniales, en éstos los paralelismos deben considerarse como "the variable outcome of constructive principles, not as fixed types of structures toward which the discourse necessarily tends" (Hanks, ibid: 93). Las cualidades retóricas de un locutor, de la cual dependen directamente su poder y eficiencia pragmática, residen en su capacidad de combinar los distintos procedimientos de una manera individualizada, a la vez estilisticamente y contextualmente. Esa necesaria parte de improvisacion contribuye también a explicar el modo de transmisión por impregnación, y jamás instrucción formal, de los discursos. Y que los recursos para la estructuración y la variación sean idénticos, hace que la densidad de recurrencia aumente con la destreza del locutor para siempre reinventar los caminos y el sentido de sus palabras, con las ondulaciones y vibraciones constantes que le dan vida.

\section{BIBLIOGRAFIA}

Breton, Alain

1994 Rabinal Achi: un drume dynostique maya du guinziène siecle. Nanterre: Société d'Ethnologie.

1999 Rabisal Acthi. Un drama dintéstico maya del sigfo XV. Guatemala: CEMCA.

Bricker, Victoria

1974 "The Ethnographic Context of Some Traditional Speech Genres", Explorations in the Ethogrophy of Speoking, pp. 368-389, R. Bauman y J. Sherzer (eds.), Cambridge: Cambridge University Press.

16 $\mathrm{A}$ very general and productive system for generating interlodking cyclical patterns in art and ritual that the Mayan peoples have exploited for ages" (Fought, 1985: 144). 
Christenson, Allen J.

2000 The Mythic Sections - Tales of First Beginnings fromt the Ancient K'iche'-Maya. EARMS. Provo, UT: Brigham Young University.

Davis, John F.

18.30 "Poesos Sinensis Comentari"., Transactions of the Royal Asiatic Society of Grear Britain and Ireiond, 2.410-19.

Fought, John

1985 "Cyclical Patterns in Chorti (Mayan) Literanura", Supplentent of the Hondbook of Middle Americont Indians, 111. Literatures, pp. 133-146, Munus S. Edmonson [ed.). Austin: University of Texas Press.

Fox, James

1975 "On Binary Categories and Primary Symbols: Some Rotinese Perspectives". The Interpretation of Symbolism, R. Willis (ed.), pp. 99-132, Londres: Malabay.

1988 To Speak in Pairs: Essuys on the Ritual Languages of Eastern Indonesia. Cambridge: Cambridge Lniversity Press.

Gossen, Gary $\mathrm{H}$.

1974 Chemules in the World of the Sun. Time and Space in a Mayo Oral Tradition. Cambridge, Mass.: Harvard University Press frad. 1979. Los chomules etr el mundo del sol. México: Instituto Nacional Indigenistal.

Hanks, William $\mathrm{F}$.

1987 "Discourse Geares in a Theory of Practice", Americant Ethnologist, 14(4): 6488 .

1986 "Authenticity and Ambivalence in the Text: A Colonia Maya Case", Ameriran Ethnologist, 13: 721-744.

1989 "Element of Maya Style", Ward and imoge in Maya Culture. Explaration in langunge, Writing, and Representation, pp. 92-111, W. E. Hanks y D. S. Rice (eds.), Salt lake City: Liniversity of Utah Press.

Haviland, john $B$.

1992 "Lenguaje ritual sin ritual", Estudios de Cultura Mayo, 19. 427-443. México: UNAM, Instituto de Investigaciones Filológicas, CIM.

Hull, Kerry $M$.

2003 "Verbal Art and Performance in Ch'orti' and Maya Hieroglyphic Writing". Ph.

D. dissertation, University of Texas at Austin.

Jakobson, Roman

1966 "Grammatical Parallelism and its Russian Facet", Lorrguoge, 42 (2) 399-429. 
Jakobson, Roman

1973 Questions de poétique. Paris: Le Seuil.

Lowth, Robert

1787 Lectures on the Sacred Poetry of the Hebrews. Londres: publicado por George Gregory.

Monod Becquelin, Autore

1979 "Examen de quelques paires sémantiques dans les dialogues rituel des Tzeltal de Bachajon flangue maya du Chapas)", Journal de la Societé des Américanites, 66: 235-263.

1986 "Le sang et le corps, ou le blanc et le noir? Contribution à l'étude du parallélisme dans la tradition orale des Maya", Journol de la Société des Antericanttes, $72: 7-31$.

1987 "Le tour du monde en quelques couplets. Le parallélisme dans la tradition orale maya". Kalevala et troditions wales du monde, pp. 467-488, J. Fernandez-Vest (ed.), París: Editions du CNRS (Colloques internationaax).

y Cédric Becquey

2008 "De las unidades paralelisticas en Las tradiciones orales mayas", Extudios de Cultura Maya, 32: 107-150. México: UNAM, Instituto de Imestigaciones Filológicas, CEM.

Montemayor, Carlos

1999 Arte y plegerie en las lenguas indigenas de México. México: FCE.

Norman, William $\mathrm{M}$.

1983 "Paralelismo gramatical en el lenguaje ritual quiche", Neves perspectivas sobre ef Popol Vuh, pp. 109-121, R. M. Carmack y E. Morales Santos (eds.). Guatemala: Editorial Piedra Santa.

Rumsey, Allan

2002 "Aspect of Ku Waru Ethnosyntax and Social Life", Ethosyntax. Explarations in Grammar and Culture, pp. 259-286. Nick J. Enfield led.). Oxford, Oxford University Press.

Tedlock, Dennis

1983a The Spoken Word and the Work of interpretation. Filadelfia: University of Pennsylvania Press.

1983b "Las formas del verbo quiche", Nirevus perspectives sobve el Popol Vuh, pp. 123-132, R. M. Carmack y E. Morales Santos [eds.). Guatemala: Editorial Piedra Santa.

Towsend, Paul G.

1983 Ritual Rhetoric from Costal. Guatemala: Instituto Linguistico de Verano. 
Vapnarsky, Valentina

1999 "Conceptions et expressions de la temporalité chez les Mayas Yucatèques (Quintana Roo)". Tesis de doctorado, Université Paris 10-Nanterre.

2000 "De dialogues en prières, la procession des mots", Les rituels du diologue, pp. 431-479, Aurore Monod Becquelin y Philippe Erikson (eds.). Nanterre: Société đEthnologie.

2001 "Cambio y continuidad en las concepciones históricas mayas : estrategias discursivas y construcción de la memoria colectiva entre los mayas macehuales de Quintana Roo", Mayu Survindism, Hostettler U. y M. Restall (eds.). Markt Schwaben, Alemania: Verlag Anton Saurwein.

2003 "Recorridos instauradores: configuración y apropiación del espacio y del tiempo entre los mayas yucatecos". Actas del Coloquio Los espacios maves: representocionies, urilizaciones y creencies. Méxicos UNAM.

"Predicción y performatividad en la memoria histótica maya yucateca", Texto $y$ contexto: Lo literatura mayo yucateca en perspectiva diocrónica. pp. 160-189, A. Gunsenheimer, T. Okoshi Harada y J. Chuchiak (eds.). Francfort: Shaker [Estudios de Americanistas de Bonn) [en prensal.

- y o. Le Guen

2006 "The Guardians of Space and History: Understanding Ecological and Historical Relations of the Contemporary Yucatec Maya to their Landscape*. ponencia presentada en Waycb, Malmö, diciembre 2006.

Woodbury, Anthony C.

1985 "Rhetorical Structure in a Central Alaskan Yupik Tradicional Narrative". Native American Discourse: Poetics and Rhetoric, pp. 1-51, Joel Sherzer y Anthony C. Woodbury (eds.). Cambridge: Cambridge University Press. 


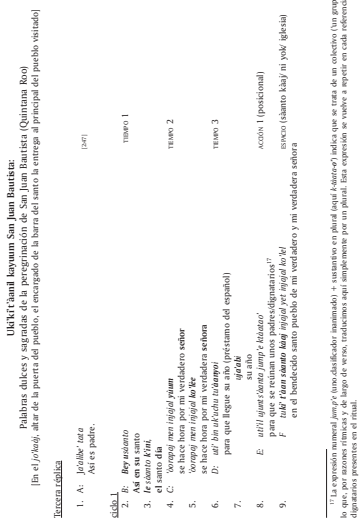




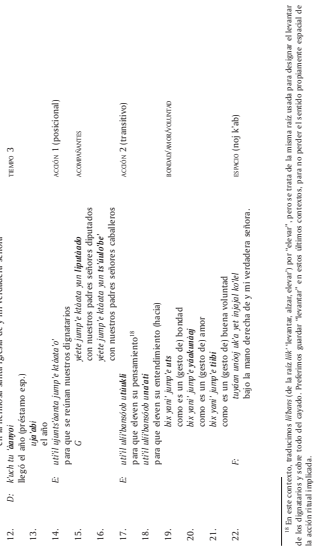



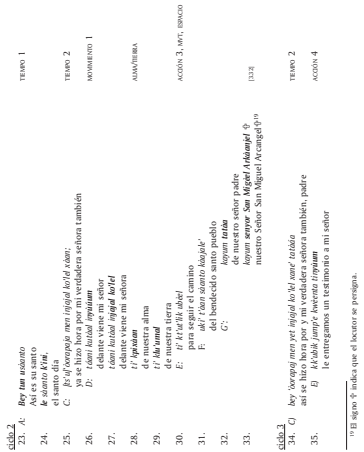


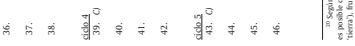



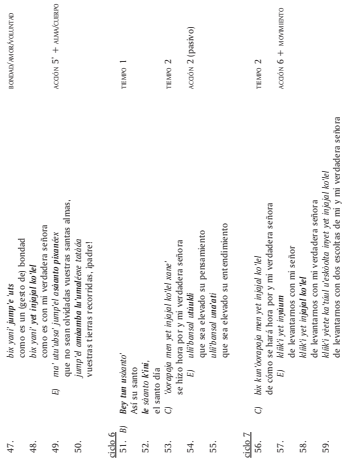


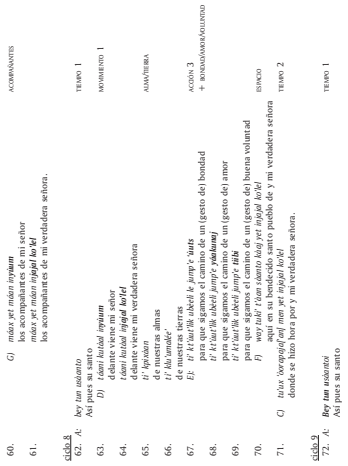



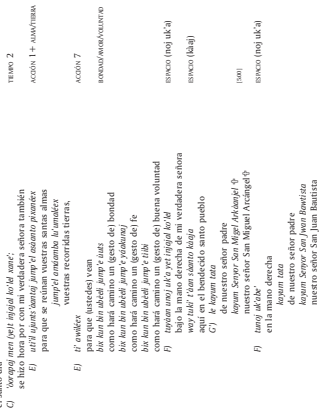

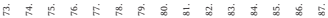



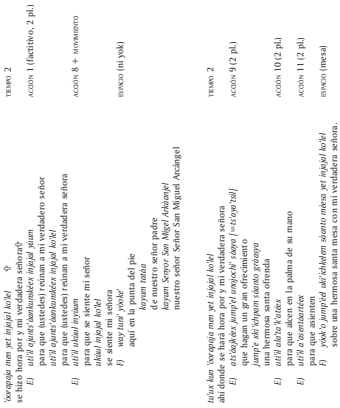

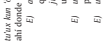

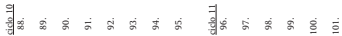



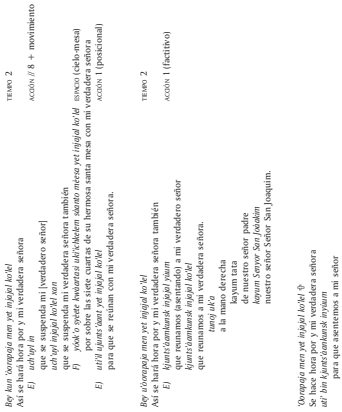

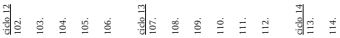




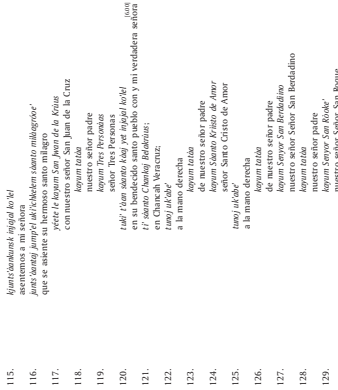




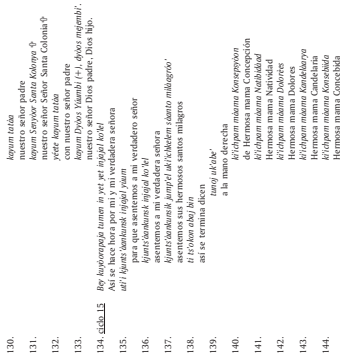




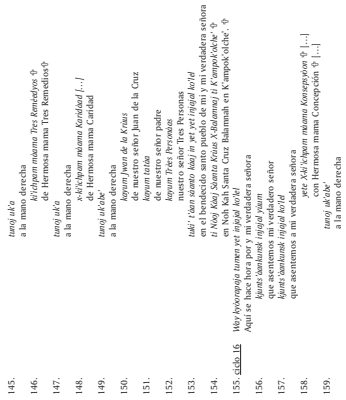




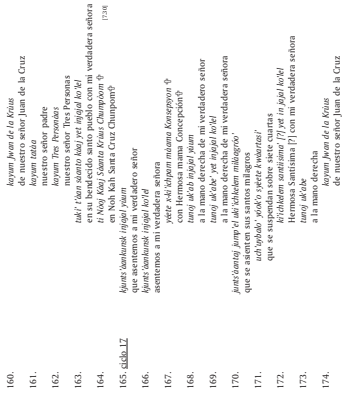




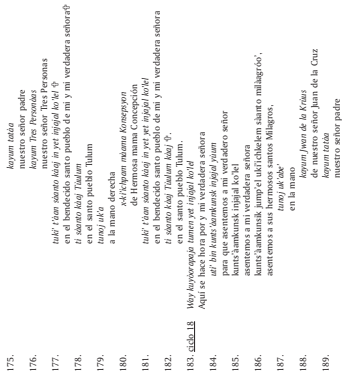




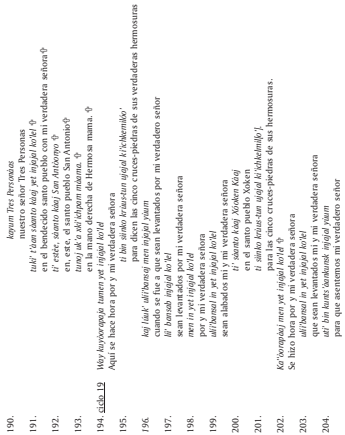




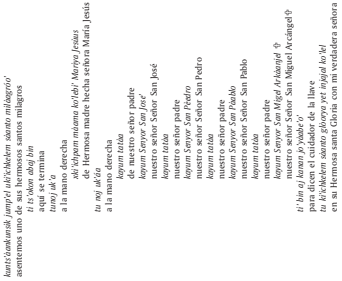

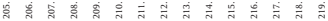




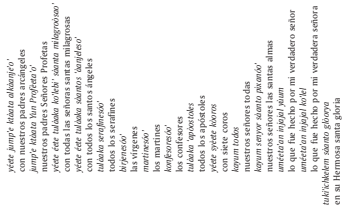

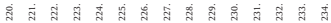




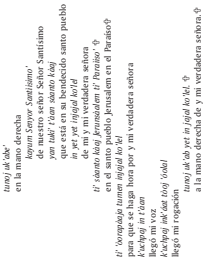

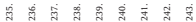

